\title{
Medial arterial calcification in the feet of diabetic patients and matched non-diabetic control subjects
}

\author{
M.J.Young ${ }^{1}$, J. E.Adams ${ }^{2}$, G.F.Anderson ${ }^{3}$, A.J.M.Boulton ${ }^{1}$, P.R.Cavanagh ${ }^{4}$ \\ ${ }^{1}$ University Department of Medicine, Manchester Royal Infirmary, Manchester, UK \\ ${ }^{2}$ University Department of Diagnostic Radiology, Manchester University Medical School, Manchester, UK \\ ${ }^{3}$ Department of Statistics, Pennsylvania State University, University Park, Pennsylvania, USA \\ ${ }^{4}$ Centre for Locomotion Studies, Pennsylvania State University, University Park, Pennsylvania, USA
}

\begin{abstract}
Summary. The prevalence and distribution of medial arterial calcification was assessed in the feet of four subject groups; 54 neuropathic diabetic patients with previous foot ulceration (U), median age 60.5 (50.5-67 interquartile range) years, duration of diabetes 19.5 (9.9-29.9) years; 40 neuropathic diabetic patients without a foot ulcer history $(\mathrm{N})$, age $68(62-$ $73)$ years, duration of diabetes $14.0(8.0-28.0)$ years; 43 nonneuropathic diabetic patients (NN), age $60.5(52-68.5)$ years, duration of diabetes $14.0(8.0-28.0)$ years and 50 non-diabetic control subjects, age 62.5 (53.7-70) years. A single radiologist graded medial arterial calcification as absent, mild or severe, at the ankle, hind-foot, mid-foot, metatarsals and toes on standardised plain lateral and antero-posterior foot radiographs taken by a single radiographer. Diabetes history, vibration perception threshold, ankle systolic pressure and serum creatinine were also assessed. Medial arterial calcification was significantly greater (total score 18 [3-31]) in neuropathic diabetic patients with previous ulceration ( $\mathrm{U}$ vs $\mathrm{N}$ $p<0.01$, U vs NN $p<0.001$ ). Non-neuropathic diabetic patients did not have significantly higher arterial calcification scores than age-matched non-diabetic control subjects. Medial arterial calcification correlated with vibration perception threshold $(r=0.35)$, duration of diabetes $(r=0.32)$ and serum creatinine $(r=0.41)$, (all $p<0.01)$. Logistic regression
\end{abstract}

models showed vibration perception and duration of diabetes to predict the probability of any calcification. Serum creatinine level was added to predict severe calcification. Ordered categorical modelling confirmed that medial arterial calcification was significantly heavier at the ankle than the toes for all groups, odds ratio $4.35(2.94-6.43,95 \%$ confidence intervals), $(p<0.01)$. Ankle systolic pressure and ankle-brachial pressure index were significantly associated with degree of arterial calcification, $r=0.40$ and $r=0.35$, respectively, (both $p<0.01$ ) in diabetic patients. However, arterial calcification was present in more than one-third of patients with an ankle-brachial pressure index of less than 1.0. In conclusion, although ankle pressures correlate with the degree of arterial calcification, medial arterial calcification may be present in patients with low ankle systolic pressures, which may be falsely elevated even at 'normal' values. This finding may provide a rationale for the use of toe rather than ankle pressure measurements in diabetic patients, particularly those with peripheral neuropathy, and this hypothesis should be directly tested in future studies.

Key words: Medial arterial calcification, neuropathy, ankle pressure index, toe pressure, screening.
Medial arterial calcification has been reported to be more frequent in patients with diabetes mellitus than in healthy subjects [1-4], and is reported to be associated with diabetic peripheral somatosensory and autonomic neuropathy [5-7]. Few previous studies have examined the distribution of medial arterial calcification quantitatively within the diabetic foot. Medial arterial calcification is significantly associated with an increased prevalence of cardiovascular mortality [7-10], although this may also be related to the increase in medial arterial calcification associated with diabetic nephropathy $[5,6]$, an independent marker of increased mortality in diabetes [11].
Peripheral vascular disease is more common in diabetic patients than healthy control subjects [10] but the relationship between medial arterial calcification and the development of clinically important peripheral vascular disease is disputed $[4,6,9,12]$. However, screening for the presence of peripheral vascular disease in diabetic patients usually involves clinical examination of the foot pulses and a measurement of the ankle brachial pressure index [13-15]. Medial arterial calcification, when present, is known to alter the pulse waveform and falsely elevate ankle pressures in diabetic patients [16, 17]. For this reason it has been suggested that toe systolic pressure meas urements might replace ankle-pressure measurements as 
Table 1. Physical characteristics of the subject groups

\begin{tabular}{|c|c|c|c|c|c|c|c|c|c|c|c|c|c|}
\hline & $n$ & $\mathrm{M}: \mathrm{F}$ & $\begin{array}{l}\text { Median } \\
\text { age } \\
\text { (years) }\end{array}$ & $\begin{array}{l}\text { Inter- } \\
\text { quartile } \\
\text { range }\end{array}$ & $\begin{array}{l}\text { VPT } \\
\text { (Volts) }\end{array}$ & $\begin{array}{l}\text { Inter- } \\
\text { quartile } \\
\text { range }\end{array}$ & $\begin{array}{l}\text { Diabetes } \\
\text { Type } \\
1: 2\end{array}$ & $\begin{array}{l}\text { Diabetes } \\
\text { duration } \\
\text { (years) }\end{array}$ & $\begin{array}{l}\text { Inter- } \\
\text { quartile } \\
\text { range }\end{array}$ & $\begin{array}{l}\mathrm{HbA}_{1} \\
(\%)^{\mathrm{a}}\end{array}$ & $\begin{array}{l}\text { Inter- } \\
\text { quartile } \\
\text { range }\end{array}$ & $\begin{array}{l}\text { Creatinine } \\
(\mu \mathrm{mol} / \mathrm{l})\end{array}$ & $\begin{array}{l}\text { Inter- } \\
\text { quartile } \\
\text { range }\end{array}$ \\
\hline $\begin{array}{l}\text { Ulcer } \\
\text { neuropathic (U) }\end{array}$ & 54 & $41: 13^{\mathrm{b}}$ & 60.5 & $50.5-67.0$ & $48.0^{\circ}$ & $40.0-51.0$ & $24: 30^{b}$ & $19.5^{\mathrm{e}}$ & $9.9-29.2$ & 10.6 & $9.1-13.1$ & $113.5^{\mathrm{e}}$ & $95.0-192.0$ \\
\hline $\begin{array}{l}\text { Neuropathic } \\
\text { no ulcer(N) }\end{array}$ & 40 & $27: 13$ & $68.0^{\mathrm{b}}$ & $62.2-73.0$ & $45.8^{\mathrm{c}}$ & $36.7-51.0$ & $16: 24$ & $14.0^{f}$ & $8.0-28.0$ & 10.6 & $9.3-11.8$ & $96.0^{\mathrm{g}}$ & $89.5-110.5$ \\
\hline $\begin{array}{l}\text { Non-neuropathic } \\
\text { (NN) }\end{array}$ & c 43 & $29: 14$ & 60.5 & $52.0-68.5$ & $17.6^{d}$ & $12.3-21.1$ & $14: 29$ & $14.0^{f}$ & $5.0-20.0$ & 10.0 & $8.4-12.0$ & $90.0^{\mathrm{g}}$ & $79.9-99.0$ \\
\hline $\begin{array}{l}\text { All diabetic } \\
\text { patients }\end{array}$ & 137 & $97: 40$ & 63.0 & $54.0-69.0$ & 40.0 & $21.8-49.9$ & $51: 86$ & 15.5 & $8.0-26.0$ & 10.5 & $9.1-12.4$ & 97.0 & $87.0-120.5$ \\
\hline $\begin{array}{l}\text { Non-diabetic } \\
\text { control subjects } \\
\text { (C) }\end{array}$ & 50 & $35: 15$ & 62.5 & $53.7-70.0$ & $15.2^{\mathrm{d}}$ & $11.0-24.2$ & & & & & & & \\
\hline All & 187 & $132: 55$ & 63.0 & $54.0-69.0$ & 34.6 & $16.4-47.6$ & & & & & & & \\
\hline
\end{tabular}

${ }^{\mathrm{a}}$ No significant difference between all groups; ${ }^{b} p<0.05 \mathrm{~N}$ vs all other groups; ${ }^{\mathrm{c}} p=\mathrm{NS} \mathrm{U}$ vs $\mathrm{N}, p<0.01 \mathrm{U}$ or $\mathrm{N}$ vs NN or C; ${ }^{d} p=\mathrm{NS} \mathrm{NN}$ vs C; ${ }^{\circ} p<0.05 \mathrm{U}$ vs $\mathrm{N}$ or NN; ${ }^{\mathrm{f}} p=\mathrm{NS} \mathrm{N}$ vs NN; ${ }^{g} p<0.05 \mathrm{~N}$ vs NN

VPT, Vibration perception threshold

an index of arterial inflow to the diabetic foot [17]. However, the accuracy of toe pressure measurements would only be superior if medial arterial calcification in the diabetic foot was less prevalent in the distal foot than the hind foot or ankle region but this has not been described in the literature.

This study examined standardised radiographs of the feet in groups of diabetic patients with and without neuropathy in a hospital-based diabetes clinic and in healthy control subjects. The purpose of the study was to determine the prevalence and distribution of medial arterial calcification in these groups and assess its implications for screening diabetic patients for lower limb peripheral vascular disease.

\section{Subjects and methods}

This study was approved by the Central Manchester Hospitals and Community Care Trust Ethical Committee and all patients and healthy volunteers gave informed consent prior to their participation.

A total of 137 diabetic patients and 50 age- and sex-matched healthy control subjects $(C)$ were included in this study. Their characteristics are detailed in Table 1 . In summary, there were 54 diabetic patients with neuropathy and a history of foot ulceration (U), 40 with neuropathy without foot ulceration (N) and 43 with no clinical neuropathy (NN). No patient had a foot ulcer at the time of study.

Diabetic subjects were chosen at random from the appropriate clinical groupings as identified on the computer database at the Manchester Diabetes Centre. In total, 179 patients were known to have had neuropathic ulceration. During 1988-1989, 520 consecutive patients had their vibration perception measured, of these 177 had a vibration perception threshold greater than $30 \mathrm{~V}$ and no history of ulceration, and 260 had a vibration perception threshold of less than $25 \mathrm{~V}$. These patients formed the patient pool for recruitment. The diabetes centre is a hospital-based diabetes clinic and, as with most studies of this type, may not be representative of the distribution of diabetes within the general population. Therefore, this study examined comparisons between groups and relationships within them rather than stating absolute prevalence rates for medial arterial calcification.

Normal non-diabetic control subjects were taken from the agesex register of a general practice within the referral area of the diabetes centre. These patients had no history of renal failure, alcoholism or other cause of neuropathy and no symptoms of intermittent claudication.

After completion of the study the diabetic neuropathic patients with previous foot ulceration were found to have a longer duration of diabetes and an excess of Type 1 (insulin-dependent) diabetic patients than the non-neuropathic diabetic patients $(p<0.05)$. Type 1 diabetic patients had an overall significantly longer duration of diabetes than the Type 2 (non-insulin-dependent) diabetic patients, median 26 (20-35 interquartile range) years vs 11 (8-19) years $(p<0.01)$. The non-ulcer neuropathic diabetic patients were older than the other diabetic groups and the non-diabetic control subjects $(p<0.05)$, (Table 1$)$.

\section{Clinical assessment}

Vibration perception threshold (VPT) was assessed by biothesiometry (Biomedical, Newbury, Ohio, USA) as the mean of the ascending threshold at five sites in each foot; the lateral malleolus, dorsum of the foot, heel, first metatarsal head and great toe. Patients were designated as neuropathic if they had a mean VPT of greater than 30 V. Foot pulses were assessed by palpation: their absence was recorded if no pulses were palpable in either foot.

The brachial systolic blood pressure was measured in the right arm and the ankle systolic pressures were measured at the dorsalis pedis artery on each foot using an handheld Doppler ultrasound (Oxford Sonicaid Ltd., Oxford, UK). The mean ankle systolic and ankle-brachial pressure index was used in the analyses.

The diabetic patients had blood taken for glycated haemoglobin $\left(\mathrm{HbA}_{1}\right.$-laboratory normal $<8 \%$ ) and serum creatinine.

\section{Radiographic technique and analysis}

Weight-bearing (standing) true antero-posterior (A-P) and lateral radiographs were taken of each foot using a standardised protocol and fine grain X-ray film and screen (Kodak Ektamat G 100 film and $\mathrm{X}$-omatic cassette with X-omatic fine intensifying screens; Kodak plc, Hemel Hempstead, Herts., UK). All the radiographs were taken by a single radiographer. True lateral radiographs were taken with a horizontal beam centred $2 \mathrm{~cm}$ above the fifth metatarsal head, the $\mathrm{X}$-ray focus-film distance (FFD) was $100 \mathrm{~cm}$, exposure $63 \mathrm{kV}$ and $25 \mathrm{~m}$.As. A-P radiographs were taken with a $20^{\circ}$ cranial tilt from the vertical at FFD $80 \mathrm{~cm}, 63 \mathrm{kV}$ and $20 \mathrm{mAs}$. Each foot was imaged separately with the beam centred on the second metatarsophalangeal 
Table 2. Prevalence of medial arterial calcification in the foot by group and region (\%)

\begin{tabular}{lrrrrrr}
\hline & $n$ & Ankle & $\begin{array}{l}\text { Hind } \\
\text { Foot }\end{array}$ & $\begin{array}{r}\text { Mid- Meta- } \\
\text { Foot }\end{array}$ & Tarsals & \\
\hline Ulcer neuropathic & 54 & 78.8 & 75.0 & 69.2 & 69.2 & 40.4 \\
Neuropathic no ulcer & 40 & 61.9 & 47.6 & 50.0 & 40.5 & 23.8 \\
Non-neuropathic & 43 & 25.0 & 29.5 & 16.0 & 22.7 & 6.8 \\
Non-diabetic controls & 50 & 22.5 & 6.0 & 10.0 & 6.0 & 0.0 \\
\hline
\end{tabular}

Table 3. Total calcification score in each subject group

\begin{tabular}{llll}
\hline & $\begin{array}{l}\text { Mean total } \\
\text { calcification } \\
\text { score }\end{array}$ & $\begin{array}{l}\text { Median total } \\
\text { calcification } \\
\text { score }\end{array}$ & $\begin{array}{l}\text { Inter- } \\
\text { quartile } \\
\text { range }\end{array}$ \\
\hline Ulcer neuropathic (U) & 18.46 & $18.0^{2}$ & $3.0-31.0$ \\
Neuropathic no ulcer (N) & 6.83 & $2.0^{\circ}$ & $0.0-13.0$ \\
Non-neuropathic (NN) & 4.3 & $0.0^{\circ}$ & $0.0-3.0$ \\
Non-diabetic controls (C) & 0.7 & 0.0 & $0.0-0.0$ \\
\hline
\end{tabular}

${ }^{\mathrm{a}} p<0.01 \mathrm{U}$ vs N; ${ }^{\mathrm{b}} p<0.01 \mathrm{~N}$ vs NN; ${ }^{\mathrm{c}} p=\mathrm{NS} \mathrm{NN}$ vs C

joint. All the radiographs were read by a single radiologist (JEA) without knowledge of the subjects' clinical category. Medial arterial calcification was defined as parallel tramline calcification and, if present, was graded as mild or severe according to previously published criteria [1]. Absent calcification was scored as 0, mild calcification as 1 and severe as 2 .

Calcification was assessed in four soft tissue regions on dorsum and plantar aspect of each lateral radiograph, these were the ankle, hind foot, mid-foot and metatarsals. As the interdigital and intermetatarsal arteries are not normally visible on lateral views, three regions on the A-P projections, the toes, metatarsals and mid-foot were also assessed.

For the purposes of analysis the calcification data were analysed in two ways. The total amount of medial arterial calcification (total arterial calcification score) was assessed as the sum of the scores in each region, giving a total maximum score of 44 if severe calcification was present in all 11 regions of each foot. Amputees were scored as twice the score of the remaining intact foot. For comparing the regions and modelling the prevalence of calcification, the presence or absence of calcification on any view in each region of either foot was used.

\section{Statistical analysis}

The majority of the data was not normally distributed and MannWhitney $U$ tests have been used throughout for the standard comparisons between groups. Chi-squared tests have been used to assess the distribution of gender and diabetes type between groups and for tests of associations. Spearman Rank correlations were used to as sess the inter-relationships between parameters. All the standard statistics were performed using Minitab software (Minitab Inc, State College, Pa., USA).

The ceiling effect of a maximum VPT of $50 \mathrm{~V}$ and a skewed distribution of calcification would artificially inflate the correlations in the diabetic groups. All correlations therefore include only those subjects with a measurable VPT and/or a minimum calcification score of 1 , this left 86 of 137 of the diabetic patients in correlation analyses.

Within the control group the total calcification score, median 0.0 (range $0-10$, interquartile range $0.0-0.0$ ) was markedly skewed to the left, with 40 of the 44 subjects scoring 0 . Therefore correlations of the total arterial calcification score and other parameters using this group were not performed.

An ordered categorical model was fit to the data [18] in order to determine the existence and nature of any ordered progression in the prevalence of calcification along the axis of the foot (ankle to toe) and between groups of patients. The presence or absence of medial arterial calcification in a given region represented the dichotomous variable of interest. The ordered categorical data analysis was performed using 'Proc Catmod' in SAS software (SAS Institute Inc., Cary, NC, USA). The complete absence of calcification in the toe region of the non-diabetic control subjects resulted in a zero cell for that data point. As this caused a singularity, which would not allow the program to run, the value 0.00001 was placed in this cell. Running the model with values as high as 0.01 did not alter the result and therefore 0.00001 was felt to be as near zero as to be insignificant in its effects on the model.

A statistical model was fit to this ordered data to reveal any trends which may have been suppressed by the underlying variability in the data. The log odds of the probability of calcification was modelled using nominal group effects $(\mathrm{U}, \mathrm{N}, \mathrm{NN}, \mathrm{C})$ and ordinal region effects (heel, hind foot, mid-foot, metatarsals, toes), with an indicator term for calcification in the toe region.

Finally, to examine the multivariate predictors of mild and severe calcification in diabetic patients, two stepwise logistical regression models were used to predict the probability of calcification in any region of the foot. In the first model patients were allocated in two groups as those with any calcification vs those with no calcification. In the second model those with severe calcification in at least one region were compared with all the others. The potential predictor variables offered to the models were ankle systolic pressure, ankle pressure index, VPT, age, sex, duration and type of diabetes, $\mathrm{HbA}_{1}$ and serum creatinine. The analysis was performed using the SAS procedure 'Proc logit'. The $p$ values for entry and exit of the model were set at 0.1 . The procedure was run with and without patients with off scale VPT $(>50 \mathrm{~V})$ and the results were similar. The results shown below are those without these patients to remove the ceiling effect.

\section{Results}

\section{Arterial calcification}

The prevalence of calcification in each region of the foot in each group of subjects is detailed in Table 2. There was a gradient in the distribution of medial arterial calcification with a greater prevalence at the ankle and hind foot than at the toes in all groups (Table 2). This is further explored in the statistical model below.

The total calcification score in the neuropathic diabetic group with a history of foot ulceration was significantly higher than the neuropathic group without foot ulceration ( $\mathrm{U}$ vs $\mathrm{N} p<0.01$ ) (Table 3 ). The neuropathic diabetic patients without foot ulceration had significantly higher total calcification scores than the non-neuropathic diabetic control subjects ( $\mathrm{N}$ vs NN $p<0.01$ ). There was no significant difference between the total calcification score in the non-neuropathic diabetic and non-diabetic groups $(p=0.25)$.

\section{Duration and type of diabetes, sex and age}

The total calcification score correlated with the duration of diabetes, $r=0.32 p<0.01$. No significant association was found between age and total arterial calcification score, either in the diabetic patients alone, $r=-0.18$ $p=\mathrm{NS}$, or in the group comprising all of the diabetic patients and control subjects, $r=-0.01 p=$ NS. 
Table 4. Systolic pressure measurements, ankle-brachial pressure indices and absent foot pulses in each group

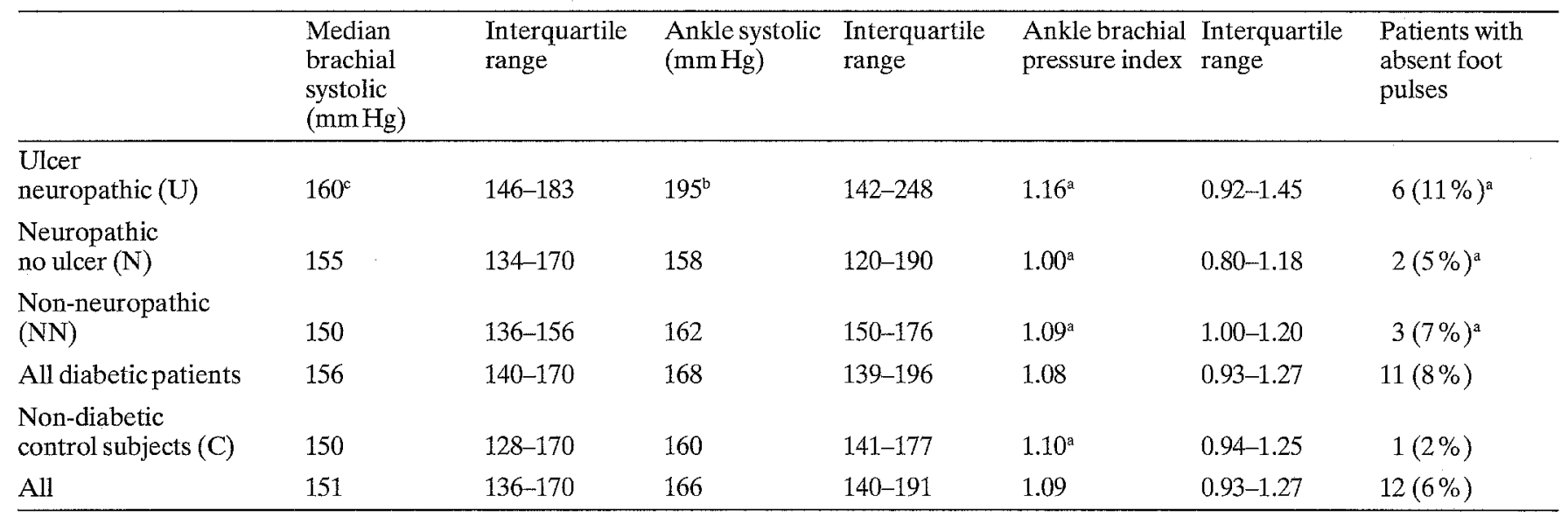

${ }^{a}$ No significant difference between all groups; ${ }^{b} p<0.05 \mathrm{U}$ vs all other groups; ${ }^{c} p=\mathrm{NS} \mathrm{U}$ vs $\mathrm{N}, p<0.01 \mathrm{U}$ vs NN or C

In the diabetic patients total arterial calcification score was significantly higher in Type 1 diabetic patients than in Type 2 diabetic patients in all groups, overall median score 13.0 (1.5-28 interquartile range) vs $1.0(0-14)$, $p<0.01$. The prevalence of medial arterial calcification was equal in Type 1 diabetic patients and Type 2 diabetic patients in each group. Type 1 diabetic patients in each group had a longer known duration of diabetes than Type 2 diabetic patients, overall median duration 20 vs 11.5 years $(p<0.01)$.

There was no significant difference in total calcification score between males and females in the diabetic groups, $4.0(0-20.5)$ vs $2.5(0-18.8), p=\mathrm{NS}$.

\section{Vibration perception threshold}

There was no significant difference between the VPT of the ulcer and non-ulcer neuropathic diabetic patients, median 48.0 (40-51 interquartile range) V vs 45.8 (36.7-51) V, $p=$ NS and no difference between the non-neuropathic diabetic and non-diabetic groups, $17.6(12.3-21.1) \mathrm{V}$ vs 15.2 (11.0-24.2) V, $p=$ NS, nor more 'off-scale' VPT readings in the neuropathic with foot ulceration group (16 vs $11, p=\mathrm{NS}$ ). By definition the neuropathic groups had significantly higher VPT than the non-neuropathic and control groups (Table 1 ).

In the diabetic patients VPT correlated with total calcification score, $r=0.35 p<0.01$. An off-scale VPT of 51 was associated with a higher prevalence of arterial calcification than a measurable VPT $(\leq 50),\left(\chi^{2}\right.$ Test, $\left.p<0.01\right)$. It predicted the presence of calcification with a specificity $96 \%$, sensitivity $29.1 \%$, positive predictive value (ppv) $92.6 \%$.

\section{Ankle and brachial systolic pressures}

The ankle and brachial pressures are detailed in Table 4 . The brachial systolic blood pressure was significantly higher in the group of neuropathic diabetic patients with a history of foot ulceration than the non-neuropathic diabetic group and the non-diabetic group but not the diabetic patients without previous foot ulceration $(p=\mathrm{NS}$ $\mathrm{U}$ vs N, $p<0.01 \mathrm{U}$ vs NN or C). The brachial systolic blood pressure did not correlate with calcification score in the diabetic patients, $r=0.09 p=$ NS.

The ankle systolic pressure was significantly higher, $(p<0.01)$, in the neuropathic diabetic patients with previous foot ulceration than in any of the other groups. None of the other groups differed significantly. The median ankle brachial pressure index did not differ significantly between the four groups. In the diabetic patients ankle systolic blood pressure correlated significantly with total arterial calcification score, $r=0.40 p<0.01$ as did the ankle brachial pressure index, $r=0.35 p<0.01$.

The specificity and sensitivity of ankle systolic blood pressure to detect the presence of medial arterial calcification is shown in Figure 1. An ankle systolic pressure of $190 \mathrm{~mm} \mathrm{Hg}$ has a $90 \%$ specificity for the presence of medial arterial calcification, but with the low sensitivity $(43.2 \%), 56.8 \%$ of patients with medial arterial calcification had ankle systolic pressures below this level. Onethird of all patients with medial arterial calcification in this series had an ankle systolic pressure below $150 \mathrm{~mm} \mathrm{Hg}$ and an ankle pressure index of less than 1.0.

The diabetic patients with a history of foot ulceration had a higher prevalence of patients with both foot pulses absent than all other groups, but this did not reach statistical significance (Table 4).

\section{Serum creatinine and $H b A_{1}$}

Total calcification score was significantly associated with serum creatinine in the diabetic patients, $r=0.41 p<0.01$. Serum creatinine was significantly higher in the ulcer neuropathic diabetic group than the other diabetic groups. The median creatinine in the non-ulcer neuropathic group was significantly higher than the non-neuropathic group (Table 1). A serum creatinine of more than $130 \mu \mathrm{mol} / 1$ was not significantly associated with an excess of medial arterial calcification ( $p=$ NS) but a creatinine greater than $150 \mu \mathrm{mol} / \mathrm{l}$ was, $(p<0.01$, sensitivity $23.5 \%$, specificity $94 \%$, ppv $87 \%$ ). 


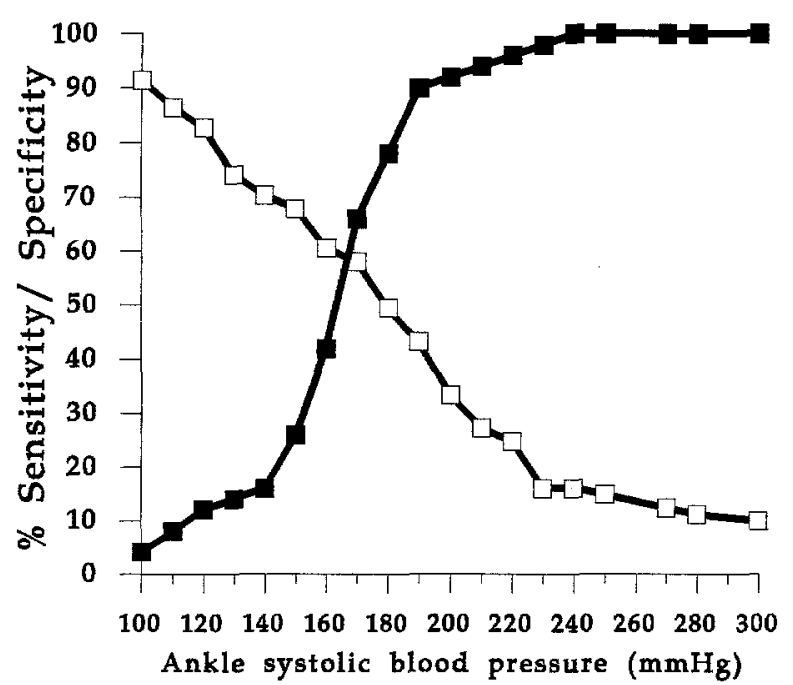

Fig. 1. Graph of sensitivity ( $\square$ ) and specificity ( $\square$ ) for the presence of medial arterial calcification for each decile of ankle systolic blood pressure

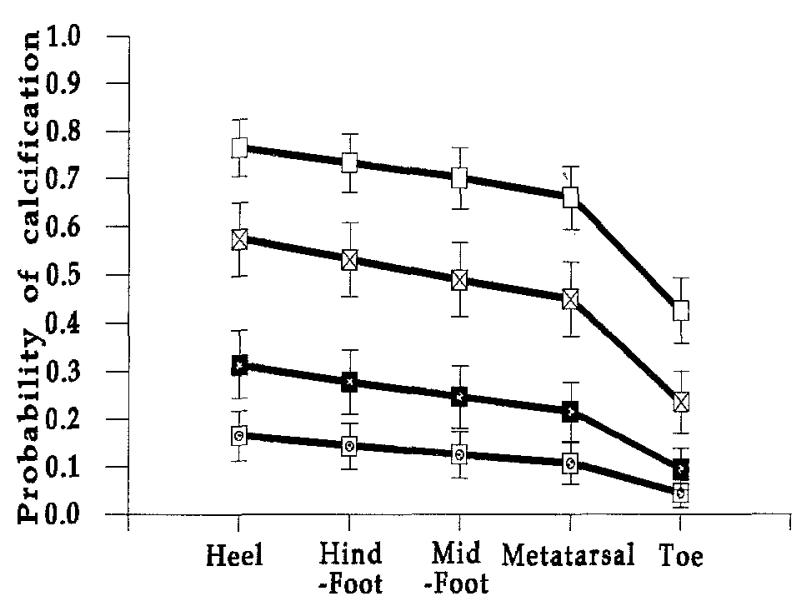

Fig.2. Predicted probability of medial arterial calcification for each subject group for each region of the foot $( \pm S E M)$ ( $\square$ Neuropathic with previous ulcer, $\otimes$ neuropathic no ulcer, non-neuropathic, [ non-diabetic control subjects)

$\mathrm{HbA}_{1}$ did not correlate with total arterial calcification score, $r=0.05 p=\mathrm{NS}$ and was not significantly different between groups (Table 1).

\section{Model of the distribution of medial arterial calcification}

The statistical model was fit to the entire data set and retained the structure of the data whilst suppressing the underlying variability. The Goodness of Fit statistic $p=0.273$ demonstrated no significant difference between the model and the data, i. e. a good fit (usual criterion $p>0.10$ ). The predicted probabilities of calcification for each region of the foot in each group are shown in Figure 2 .

A significant difference in odds of calcification was found between neuropathic diabetic patients with and without previous foot ulceration $(p<0.01)$. There was no significant difference between the predicted probability of calcification in non-neuropathic patients than in nondiabetic control subjects $(p=0.09)$. Neuropathic diabetic patients had a significantly higher probability of calcification than non-neuropathic (diabetic or control) patients $(p<0.001)$.

There was a significant association between odds of calcification and foot region $(p<0.001)$. The odds of calcification were found to increase by a multiple of 1.16 (1.07$1.26,95 \%$ confidence intervals) for each region nearer the ankle. In addition to this ordered effect of region, a significant toe effect was noted $(p<0.0001)$ which decreased the odds of calcification by an additional factor of 0.42 . Overall, the predicted odds of calcification in the ankle were 4.35 (2.94-6.43) times greater than in the toes $(p<0.001)$.

\section{Multiple logistic regression}

The presence of any calcification, either mild or severe, in a patient was predicted by two variables, VPT and duration of diabetes, where

$\log$ odds $[\mathrm{P}($ calcification $)]=-2.943+0.069 * \mathrm{VPT}+$ $0.060 *$ duration

This indicated that a $5 \mathrm{~V}$ increase in VPT increased the log odds of the presence of any calcification in the foot by a multiple of 1.42 (calculated from $\exp \{5 * 0.069\}$ ) and a 5year increment in diabetes duration increased the $\log$ odds of calcification by a multiple of 1.34 (exp. $\left\{5^{*}\right.$ $0.060\}$ ).

The presence of severe calcification in at least one region of the foot could be modelled using three variables, VPT, duration of diabetes and serum creatinine.

$\log$ odds $[\mathrm{P}($ calcification $)]=-4.841+0.053 * \mathrm{VPT}+$ $0.076 *$ duration $+0.012 *$ creatinine

By a similar analysis of the individual coefficients as shown in the previous model, a $5 \mathrm{~V}$ increase in VPT increased the log odds of the presence of severe calcification in any region of the foot by a multiple of 1.3 , a 5-year increment in diabetes duration increased the log odds of calcification by a multiple of 1.46 and a $30 \mu \mathrm{mol} / / \mathrm{l}$ increase in serum creatinine increased the log odds of calcification by a multiple of 1.42 .

\section{Discussion}

This study describes a significant association between diabetes, peripheral neuropathy and the presence of medial arterial calcification. There is also a significant gradient in the prevalence of medial arterial calcification from the ankle to the toe. The association of medial arterial calcification with diabetes [1-4] and neuropathy [5-7] has been described previously but there have been no previous studies of groups with different levels of diabetic complications or with age- and sex-matched normal con- 
trol subjects using a standardised radiographic technique for each patient to allow for accurate comparisons between regions and groups.

The development of medial arterial calcification is believed to be due to autonomic denervation of the intima media of the small muscular arteries of the foot [5-7], and increased medial arterial calcification has been reported following sympathectomy [6]. Edmonds et al. [5] described increased vascular calcification in the knee, the hands and the feet in diabetic patients with autonomic and peripheral sensory neuropathy. In the present study the total amount of medial arterial calcification was significantly higher in the neuropathic diabetic groups than in the non-neuropathic diabetic group. The total arterial calcification score also correlated significantly with the vibration perception threshold and provides quantitative confirmation of this hypothesis.

Uncomplicated diabetes, as represented in the nonneuropathic diabetic group, did not appear to increase the development of medial arterial calcification when compared with the non-diabetic group of a similar age. This was apparent from both the total calcification score and from the statistical modelling of the calcification data but no previous study has compared uncomplicated diabetic patients with normal control subjects to confirm this [1$10]$.

The increase in medial arterial calcification from nonneuropathic diabetic control subjects through neuropathic patients without a history of foot ulceration to neuropathic diabetic patients with a history of foot ulceration is probably due to a combination of factors. The addition of peripheral neuropathy is associated with a significant increase in the probability of the presence of calcification and the total amount of arterial calcification, despite the fact that the non-neuropathic and neuropathic non-ulcer diabetic patients have a very similar duration of diabetes. The neuropathic group with previous foot ulceration had a higher serum creatinine and a longer duration of diabetes than the non-ulcer neuropathic group. VPT was not significantly different between these groups and this may be because of the proximity to the ceiling of measurable VPT and the large number of 'off-scale' values in each group, 16 of 54 and 11 of 40, respectively, although the median and interquartile range were higher in the ulcer group. A chi-square test of presence of calcification with an off-scale VPT was also highly significant, further reinforcing the association of medial arterial calcification and neuropathy.

The combination of factors, serum creatinine, duration of diabetes and VPT, all of which significantly correlated independently with the total calcification score and strongly predicted the presence of severe calcification in the multiple logistic regression model, may have contributed to the higher levels of arterial calcification within the ulcer neuropathic group. It is not however possible, from the present analysis to infer that the three predictor variables are independent risk factors for the probability of the presence of severe arterial calcification.

The lack of any correlation with age and calcification is surprising. Increasing age is associated with an increase in medial arterial calcification in a number of studies $[1-4,6]$.
A possible explanation is the high median and narrow range of age of our subjects. Three-quarters were over 50 years of age, at which time calcification was more common in all these studies. This may have masked any possible relationship of calcification with age. However, the non-diabetic control group were well matched to the diabetic group in age and yet they had virtually no arterial calcification.

The higher total arterial calcification score in Type 1 diabetic patients in each group is probably due to the longer known duration of diabetes in these patients compared to Type 2 diabetic patients. This is in keeping with a report of higher ankle-brachial pressure indices in Type 1 diabetic patients which was attributed to higher levels of medial arterial calcification and a longer duration of diabetes [19]. This was not reflected in an increase in the prevalence of any degree of calcification in Type 1 diabetic patients as demonstrated by the fact that diabetes type did not enter the logistic regression model.

The probability of calcification in each group suggested a trend from non-neuropathic diabetic patients to ulcer diabetic patients and from the toe to the ankle region in each group. The ordered categorical model [18] allows further analysis of repeated ordinal measurements by suppressing the underlying variability of these measurements. The predicted probability of calcification confirmed the apparent higher probability of medial arterial calcification in neuropathic diabetic patients with previous foot ulcers than in neuropathic diabetic patients without a history of foot ulceration. It also confirmed the increase in calcification with neuropathy and also that diabetes alone, when not complicated by neuropathy, did not add to the prevalence of calcification.

The ordered effect of region, which represents the increase in calcification progressing from the metatarsals to the ankles provided a good linear fit of predicted prevalence vs observed prevalence. However, the prevalence of medial arterial calcification at the toe in all groups was significantly lower than that predicted by a purely linear model. This 'toe effect' was estimated and when introduced as a factor of 0.42 times the odds of calcification for a linear fit, made the model more representative of the data.

The testing of the raw prevalence data with a statistical model has confirmed the inter-group and inter-regional trends which would have been difficult to test significantly using individual statistical tests. These trends are also clinically relevant.

Screening diabetic patients for peripheral vascular disease and implied cardiovascular disease [4, 6, 9, 13-15] often includes a measurement of the ankle brachial systolic pressure index [13-15]. This measure has however been discredited by the false elevation reported in diabetic patients with non-compressible medial arterial calcification in the tibial and ankle plexus arteries $[16,17]$. Ankle systolic pressure and ankle-brachial pressure index were significantly associated with total arterial calcification score in this study, and, as calcification was more marked in the ankle and hind foot regions this would support the assumption. However, whilst an ankle systolic blood pressure of $200 \mathrm{~mm} \mathrm{Hg}$ or more is often believed to 
demonstrate the presence of medial arterial calcification, this study demonstrates that arterial calcification is present in $8.6 \%$ of patients with ankle systolic pressures below $100 \mathrm{~mm} \mathrm{Hg}$. Carter [20], investigated the effect of medial arterial calcification on intra-arterial measurements of dorsalis pedis arterial pressure in five patients. One patient had a significant rise in cuff pressure vs directly measured arterial pressure, whilst the others had slightly lower cuff pressures. Similar results were found in patients with brachial artery calcification. However, this study was uncontrolled and requires further studies to be performed to confirm the main findings, particularly as they appear to contradict clinical and non-invasive testing experience $[7,15-17,19]$. It is therefore quite likely that in diabetic patients ankle systolic pressures and ankle-pressure indices may be falsely elevated even at 'normal' levels due to medial arterial calcification. For this reason, toe systolic pressure is regarded as a more reliable indicator of arterial inflow to the foot than ankle pressure in diabetic patients [17]. The demonstration of significantly lower levels of arterial calcification in the toe regions provides a possible explanation for this premise and a rationale for the use of toe, rather than ankle, pressure measurements in diabetic patients. However, this hypothesis remains to be tested.

In conclusion, this study, using standardised methods to compare well-defined groups, has confirmed the previous assumptions about the associations of medial arterial calcification and neuropathy, duration of diabetes and renal dysfunction. The low discriminatory value of ankle pressures to detect arterial calcification and the converse, that calcification may be elevating the ankle-pressure index, even in the normal range, must cast further doubt over the use of ankle pressures to screen for lower limb arterial disease. The demonstration of a gradient in calcification between the ankle and the toes supports the theoretical use of toe pressure measurements rather than ankle pressures in diabetic patients, particularly those with neuropathy, but this benefit remains to be proven clinically.

Acknowledgements. We would like to thank Ms K. L. Vickers for her work in taking all the radiographs and Dr. A. Robertson, Denton, Manchester, UK for allowing us to approach his patients to participate as control subjects. Dr. M. Young is supported by the Peter Kershaw Trust. Professor Cavanagh was Visiting Professor, University Department of Medicine, Manchester Royal Infirmary, when this work was performed.

\section{References}

1. Morrison LB, Bogan IK (1929) Calcification of the vessels in diabetes. JAMA 92: 1424-1426

2. Strandness DE Jr, Priest RE, Gibbons GE (1964) Combined clinical and pathologic study of diabetic and non diabetic peripheral arterial disease. Diabetes 13: 366-372
3. Ferrier TM (1964) Radiologically demonstrable arterial calcification in diabetes mellitus. Australasian Ann Med 13:222-228

4. Neubauer B (1971) A quantitative study of peripheral arterial calcification and glucose tolerance in elderly diabetics and nondiabetics. Diabetologia 9: 409-413

5. Edmonds ME, Morrison N, Laws JW, Watkins PJ (1982) Medial arterial calcification and diabetic neuropathy. BMJ 284: 928-930

6. Goebel F-D, Fuessi HS (1983) Mönckeberg's sclerosis after sympathetic denervation in diabetic and non-diabetic subjects. Diabetologia 24: 348-350

7. Everhart JE, Pettitt DJ, Knowler WC, Rose FA, Bennett PH (1988) Medial arterial calcification and its association with mortality and complications of diabetes. Diabetologia 31: 16-23

8. Lachman AS, Spray TL, Kerwin DM, Shugoll GI, Roberts WC (1977) Medial calcinosis of Mönckeberg. A review of the problem and a description of a patient with involvement of peripheral, visceral and coronary arteries. Am J Med 63:615-622

9. Nillson SE, Lindholm H, Bülow S, Frostberg N, Emilsson T, Stenkula G (1967) The Kristianstad survey 63-64 (calcifications in arteries of lower limbs). Acta Medica Scand 428 [Suppl]: 1-46

10. Janka HU, Stadl E, Mehnert H (1980) Peripheral vascular disease in diabetes mellitus and its relation to cardiovascular risk factors: screening with Doppler ultrasonic technique. Diabetes Care 3: 207-213

11. Jensen T, Borch-Johnsen K, Kofoed-Enevoldsen A, Deckert T (1987) Coronary heart disease in young type 1 (insulin-dependent) diabetic patients with and without diabetic nephropathy: incidence and risk factors. Diabetologia 30: 144-148

12. Chantelau E, Ma XY, Herrnberger S, Dohmen C, Trappe P, Baba $\mathrm{T}$ (1990) Effect of medial arterial calcification on $\mathrm{O}_{2}$ supply to exercising diabetic feet. Diabetes 39: 513-516

13. Carter SA (1969) Clinical measurement of systolic pressures in limbs with arterial occlusive disease. JAMA 207: 1869-1872

14. Yao ST, Hobbs JT, Irvine WT (1969) Ankle systolic pressure measurements in arterial disease affecting the lower extremities. Br J Surg 56: 676-679

15. Cutajar CL, Marston A, Newcombe JF (1973) Value of cuff occlusion pressures in assessment of peripheral vascular disease. BMJ 2: 392-395

16. Reimann $H_{1}$ Bollinger A (1974) Pseudohypertonie bei Mediasklerose. Schweiz Med Wochenschr 104: 1813-1817

17. European Consensus Document on Critical Limb Ischaemia (1989) Dormandy J (ed), Springer Verlag, Berlin

18. Agresti A (1990) Categorical data analysis. John Wiley and Sons, New York

19. Goss DE, de Trafford J, Roberts VC, Flynn MD, Edmonds ME, Watkins PJ (1989) Raised ankle/brachial pressure index in insulin-treated diabetic patients. Diabetic Med 6: 576-578

20. Carter SA (1973) The relationship of distal systolic pressures to healing of skin lesions in limbs with arterial occlusive disease, with special reference to diabetes mellitus. Scand J Clin Lab Invest 31 [Suppl 128]: 239-243

Received: 15 December 1992

and in revised form: 23 February 1993

Dr. A. J.M. Boulton

Department of Medicine

Manchester Royal Infirmary

Oxford Road

Manchester M13 9WL

UK 